\title{
Precise alignment and permanent mounting of thin and lightweight $X$ - ray segments
}

\author{
Michael P. Biskach* ${ }^{* a}$, Kai-Wing Chan ${ }^{\text {b, }}$, Melinda N. Hong ${ }^{a}$, James R. Mazzarella ${ }^{a}$, Ryan S. \\ McClelland ${ }^{a}$, Michael J. Norman ${ }^{a}$, Timo T. Saha ${ }^{d}$, William W. Zhang ${ }^{c}$ \\ ${ }^{2}$ SGT Inc., 7701 Greenbelt Road, Suite 400, Greenbelt, MD, USA 20770. \\ ${ }^{\mathrm{b}}$ Center for Research and Exploration in Space Science \& Technology, Univ. of Maryland, \\ Baltimore County, 1000 Hilltop Cir., Baltimore, MD, USA 21250 \\ ${ }^{c}$ X-ray Astrophysics Laboratory, NASA Goddard Space Flight Center, Greenbelt, MD, USA 20771 \\ 'Optics Branch, NASA Goddard Space Flight Center, Greenbelt, MD, USA 20771
}

\begin{abstract}
To provide observations to support current research efforts in high energy astrophysics, future X-ray telescope designs must provide matching or better angular resolution while significantly increasing the total collecting area. In such a design the permanent mounting of thin and lightweight segments is critical to the overall performance of the complete $\mathrm{X}$-ray optic assembly. The thin and lightweight segments used in the assembly of the modules are designed to maintain and/or exceed the resolution of existing X-ray telescopes while providing a substantial increase in collecting area. Such thin and delicate X-ray segments are easily distorted and yet must be aligned to the arcsecond level and retain accurate alignment for many years. The Next Generation X-ray Optic (NGXO) group at NASA Goddard Space Flight Center has designed, assembled, and implemented new hardware and procedures with the short term goal of aligning three pairs of $\mathrm{X}$-ray segments in a technology demonstration module while maintaining $10 \operatorname{arcsec}$ alignment through environmental testing as part of the eventual design and construction of a full sized module capable of housing hundreds of X-ray segments. The recent attempts at multiple segment pair alignment and permanent mounting is described along with an overview of the procedure used. A look into what the next year will bring for the alignment and permanent segment mounting effort illustrates some of the challenges left to overcome before an attempt to populate a full sized module can begin.
\end{abstract}

Keywords: X-ray optics, segmented optics, optical alignment, permanent mounting, co-alignment

\section{INTRODUCTION}

The field of high energy astrophysics, specifically observations in the X-ray region of the electromagnetic spectrum, is a rich source of information applicable to several fundamental areas of research. The origins of stars and galaxies, the study of active galactic nuclei, black holes, neutron stars, the nature of dark matter and dark energy are all areas of active research that directly benefit from observations from X-ray telescopes such as XMM-Newton and Chandra.

\subsection{Future X-ray telescope design goals}

The total collecting area for telescope designs such as XMM-Newton $\left(0.47 \mathrm{~m}^{2}\right.$ at $\left.1 \mathrm{keV}\right)$ and Chandra $\left(0.04 \mathrm{~m}^{2}\right.$ at $\left.1 \mathrm{keV}\right)$ limits the observations of many transient phenomena such as relativistic reverberation of $\mathrm{X}$-ray sources near the event horizon of a black hole ${ }^{1}$. A desirable future X-ray telescope would be one that meets or exceeds the angular resolution of current X-ray telescopes with a significant increase in total collecting area and an acceptable telescope mass and therefore cost. A possible future X-ray telescope of the typical Wolter I optic design like that of XMM-Newton and Chandra would therefore require a far greater number of reflecting surfaces using thinner segments.

\subsection{Next generation Flight Mirror Asembly (FM A) concept}

Lightweight and thin X-ray segments utilized by the Next Generation X-ray Optics (NGXO) group at NASA Goddard Space Flight Center are manufactured using commercially available flat pieces of glass (Schott D264 at $0.4 \mathrm{~mm}$ 
*michael.biskach@nașa.gov; phone 1301 286-1396;

thickness). These flat pieces of glass are individually slumped in an oven at an elevated temperature for a period of time over highly polished precision mandrels. Once the slumping process is complete, the previously flat piece of glass is now round and shaped precisely to focus X-rays ${ }^{2}$. The newly formed segment is then integrated into a module containing hundreds of closely nested segments. Multiple modules are aligned and mounted into a single Flight Mirror Assembly (FMA) that constitutes the optics of the X-ray telescope ${ }^{3}$. Each of the thousands of segments mounted in the numerous modules direct $\mathrm{X}$-rays to a common focal point (see Figure 1.

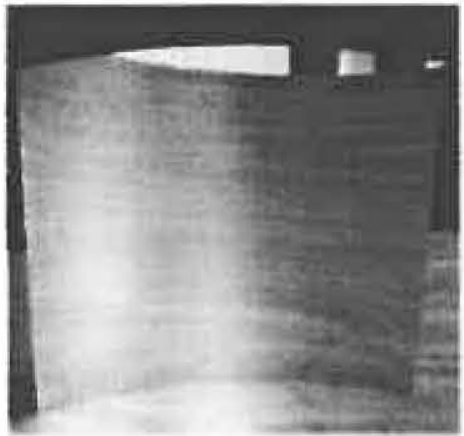

Mirror Segment

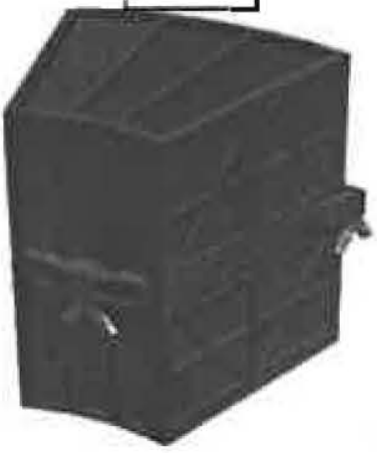

Mirror Module

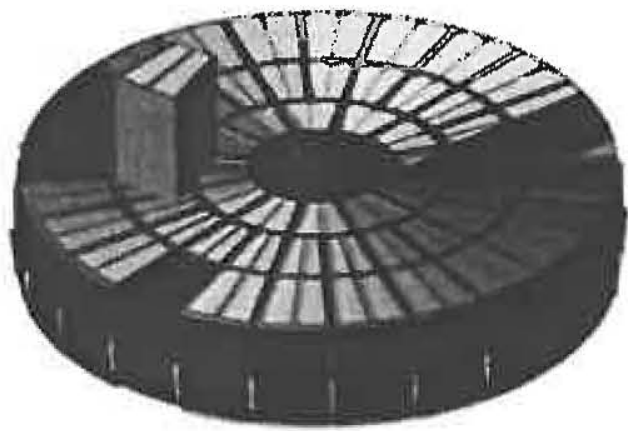

Telescope

Figure 1. Modules containing hundreds of precisely formed mirror segments are integrated into the flight mirror assembly (FMA). X-rays that reflect off of the thousands of individual mirror segments are focused to set of onboard instruments and detectors.

\subsection{Challenges in aligning and permanent mounting of segments}

As segment thickness decreases, numerous effects on segment surface accuracy and quality affect overall resolution. Gravitational effects, thermal gradients, mounting effects, etc all have some affect on the overall optical performance. An additional factor for FMA construction using thinner segments is the cost of aligning and mounting additional segments. The process for which individual segments are aligned and permanently mounted into modules must be robust, repeatable, parallel, efficient and affordable. Assembly of a module will take a certain amount of time to complete, in which every segment must retain alignment with respect to other segments (co-alignment). The permanent mounting process for each segment is required to maintain correct alignment throughout module construction, FMA integration, telescope transportation and launch, and throughout the life of the mission which may last for a decade or more. The permanent mounting process must be sufficiently strong to meet these strict mission requirements but not adversely affect the arc-second precision of the individual segment.

\subsection{Introduction to segment alignment and permanent mounting}

After each mirror segment is slumped, coated with a reflective layer, and measured to ensure surface shape and quality the segment is prepped for alignment into a module. Once the segment is prepped for alignment, the segment can then be treated as a rigid body and manipulated by a 6 degree of freedom (DOF) hexapod capable of submicron movements. Alignment measurements are performed on the mirror that is temporarily held by the hexapod and compared to segments that are already permanently mounted in a module. Slight adjustments to the segment position and angle down to the micron and arc-second level can be made using the hexapod until the segment is aligned to the existing mirrors. A permanent segment mounting process known as "edge-bonding" is used to mount the segment into the module at six locations, three on each side edge of the segment while minimizing segment mounting distortions. After adhesive used to permanently mount the segment has cured enough to support the mirror, the hexapod that temporarily supports the segment is removed and ready to support the next segment to be aligned and permanently mounted. This paper will outline the details of the segment alignment and permanent mounting process, identify current challenges, present results collected so far for the first attempts of segment integration, and discuss the next steps necessary to continue the development of a mission capable alignment and permanent mounting process. 


\section{MIRROR SEGMENT ALIGNMENT AND PERMANENT MOUNTING PROCESS}

\section{M irror alignment and per manent mounting process overview}

The main steps required to align and permanently mount a segment were briefly introduced in Section 1.4 and are as follows:

1. Temporary mounting of a segment to a precision $6 \mathrm{DOF}$ hexapod

2. Measurement of the segment alignment and calculation of the alignment half-power diameter (HPD)

3. Use of the hexapod to align the segment

4. Permanent mounting of the segment to the module

5. Removal of the temporary mount of the hexapod

\subsection{Temporary mounting of a single mirror}

Temporary mounting of a segment needed to manipulate the segment as a rigid body during alignment is a challenge by itself. Any temporary mounting of a segment must:

1. minimize segment distortions (whether the distortions are mount induced or gravitational in nature)

2. securely mount the segment to prevent shifting in alignment before or during permanent mounting (this includes mechanical drifting, thermal changes, etc)

3. allow segment to be manipulated as rigid body

4. not add significant time and complexity to segment alignment and permanent mount process

Requirements 1 and 2 may seem contradictory, the segment must be held securely but external forces can add distertions to the mirror. As a result any temporary distortions can affect the final alignment during temporary mount removal.

By limiting the number of temporary constraints applied to the segment to six, over-constraints that may cause unwanted segment distortions are no longer an influence on the final segment alignment. The kinematic nature of a temporary mount has several other important advantages including thermal insensitivity and segment placement repeatability down to the arc-second. Using Finite Element models of a segment, it was found that two mount points on the bottom edge of the segment and one at the top edge minimized gravitational distortions in the segments. The segment geometry did not allow for a straightforward kinematic temporary mount at these optimized segment locations so small steel balls $2.4 \mathrm{~mm}$ in diameter were temporarily bonded to the top and bottom edges of each segment. The kinematic balls mounted directly to the segment act as kinematic mount points. A separate structure complete with a socket and a slot for the two bottom kinematic balls to rest in and an upper plate for the top kinematic ball to rest against could then be attached to a 6 DOF hexapod without significant segment distortion. After a segment is aligned and permanently mounted into a module, the kinematic balls which are bonded onto the segment with a minimal amount of quick cure epoxy can be carefully removed since the temporary segment mount is no longer needed. Time needed to attach the kinematic balls is not a factor as the whole process typically takes 15 minutes per segment to apply quick cure epoxy to the top and bottom mirror edges and stick the three kinematic balls in place and for the epoxy to harden. The kinematic temporary therefore mount satisfies all four major requirements of temporary segment mount.

\section{Hartmann alignment measurement}

Alignment and permanent mounting of thousands of segments in X-ray only is currently not feasible. Instead, alignment and permanent segment mounting are performed using visible light to reflect off of the segments into a standard CCD positioned at the focal point of the module. After alignment and permanent segment mounting is completed using optical light, the final performance of all segments are then verified in X-ray.

To verify segment alignment in optical light, a process known as a Hartmann measurement is used. A moveable mask blocks except for a 2 degree wide axial slice of the segment. Figure 2 shows how a series of images are captured sequentially as the moveable Hartmann mask scans across the segment from one side to the other. For each image in the set, a center of intensity (Hartmann centroid) is calculated. The centroids that are calculated for the set of images are then plotted as one Hartmann measurement on an XY graph. The shape of the centroid graph and how close the 
centroids are to each other determine how well the segment is aligned. The diameter of a circle that can enclose the half of the centroids closest to the center of the XY centroid graph is known as the Half Point Diameter (HPD) and has angular units (typically arc-seconds). One of the requirements of the alignment of a segment is that the HPD value is minimized (the segment focus is optimized). The information from a Hartmann measurement is powerful and is used to optimize a new segment position, compare co-alignment between two segments, and track long term changes in a permanently mounted segment.

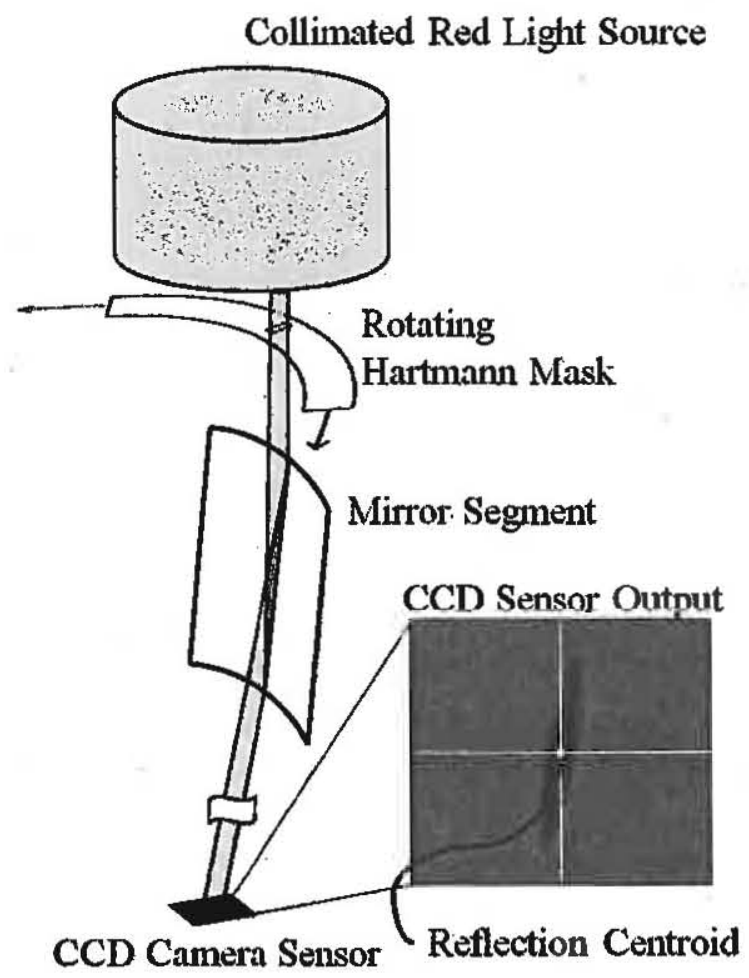

Figure 2. As the Hartmann mask rotates from one side of the segment to the other, images are taken using a CCD Camera at the focal point of the segment(s). The set of centroids calculated from the set of Hartmann images are then used to calculate an alignment HPD (in arcseconds) which determines the quality of the segment alignment.

\section{Alignment of a single segment pair}

A second moveable mask used to block desired pairs (pair selection mask) is mounted to a linear stage between the light source used to align segments and the module that will eventually house all mirror segments. This mask is aligned precisely to the module in the roll, lateral, and radial directions. Therefore the pair selection mask is typically used to align new segments in roll, lateral, and radial directions. The module will also directly determine the vertical position of a segment. Hartmann measurements determine the pitch and yaw of a single segment pair such that the HPD is minimized. Altogether the segment lateral, vertical, radial, pitch, roll, and yaw are determined from the pair selection mask (aligned to the module), the module itself, or the HPD (as calculated by the Hartmann measurement). The 6 DOF hexapod is used to make the fine adjustment in lateral, vertical, radial, pitch, roll, and yaw. The only difference between aligning a primary segment versus a secondary segment is that the radial position of the secondary is determined by maximizing the amount of visible light that is reflected off of both the primary and secondary segment surfaces (maximizing segment pair collecting area).

\section{Co-alignment of multiple mirror pairs}

Once a single segment or segment pair is permanently mounted in a module, the subsequent segments must align to the same location as the existing segment(s). A Hartmann measurement for previously mounted segments is one way to determine the location of the reflection at the focal point (also corresponds to the location of the optical CCD). The 
average of the Hartmann centroids offers a means to locate a segment reflection relative to the segment reflections of previously mounted segments.

Take an instance where there are permanently mounted segments in a module. Hartmann measurements confirm the location each segment reflection to be at the same point to within a small error (known as the co-alignment error). A new segment that is temporarily mounted on a hexapod is then aligned using the typical alignment procedure. It is possible that the new segment has an optimized HPD but the location of the reflection does not match the location of the permanently mounted segment reflections. At this point there are several corrections that can be made to co-locate the reflections from permanently mounted segments and temporarily mounted segments. If the reflection is vertically separated from the existing segment reflections, a pitch adjustment and be made to co-align the reflections. If the reflections are horizontally separated, a roll adjustment can be made to co-align the reflections. In some cases adjustments made to improve co-alignment may adversely affect the single segment (or segment pair) HPD. The change in HPD is relatively small and necessary to avoid large $(>1 \mathrm{arcsec})$ co-alignment errors between segments.

\section{Permanent mounting process for a single mirror (Edge-bond)}

For a permanent segment mounting process to be considered successful it must accomplish the following:

1. Avoid distorting the segment throughout the process

2. Maintain alignment and co-alignment with other bonded mirrors in the module

3. Maintain long term stability over the course of days, months, years, decades

4. Tens of thousands of permanent bonds to complete an FMA calls for a robust and repeatable permanent mounting process

5. To keep mission costs to an acceptable level, the permanent mounting process must be relatively fast $(\sim 1-2$ segments permanently mounted per day per module)

6. Duplicable to allow parallel module integration, therefore equipment and processes must be able to be copied and work consistently for building up multiple modules at an acceptable cost

One permanent mounting process that is currently under development is known as Edge-bonding. This is a three step process that permanently mounts a segment into a module by six bonds, three bonds on each side of a segment. The segments are less sensitive to potential enforced displacements along the azimuthal direction as shown by ray trace simulations of distorted FEA segment models.

Each segment bonded into a module is prepared before the alignment and permanent mounting process can begin. The segment preparation steps include segment slumping, coating, and reflecting surface metrology measurements to verify acceptable segment shape. At this point the segment undergoes the first of three bonds that will eventually complete the Edge-bond process. The three bonds in Edge-bonding are referred to as: P0 (clips to segment bond), P1 (pin to clip bond and P2 (pin to module bond). See Figure 3 for a summary of the three stages of Edge-bonding. 


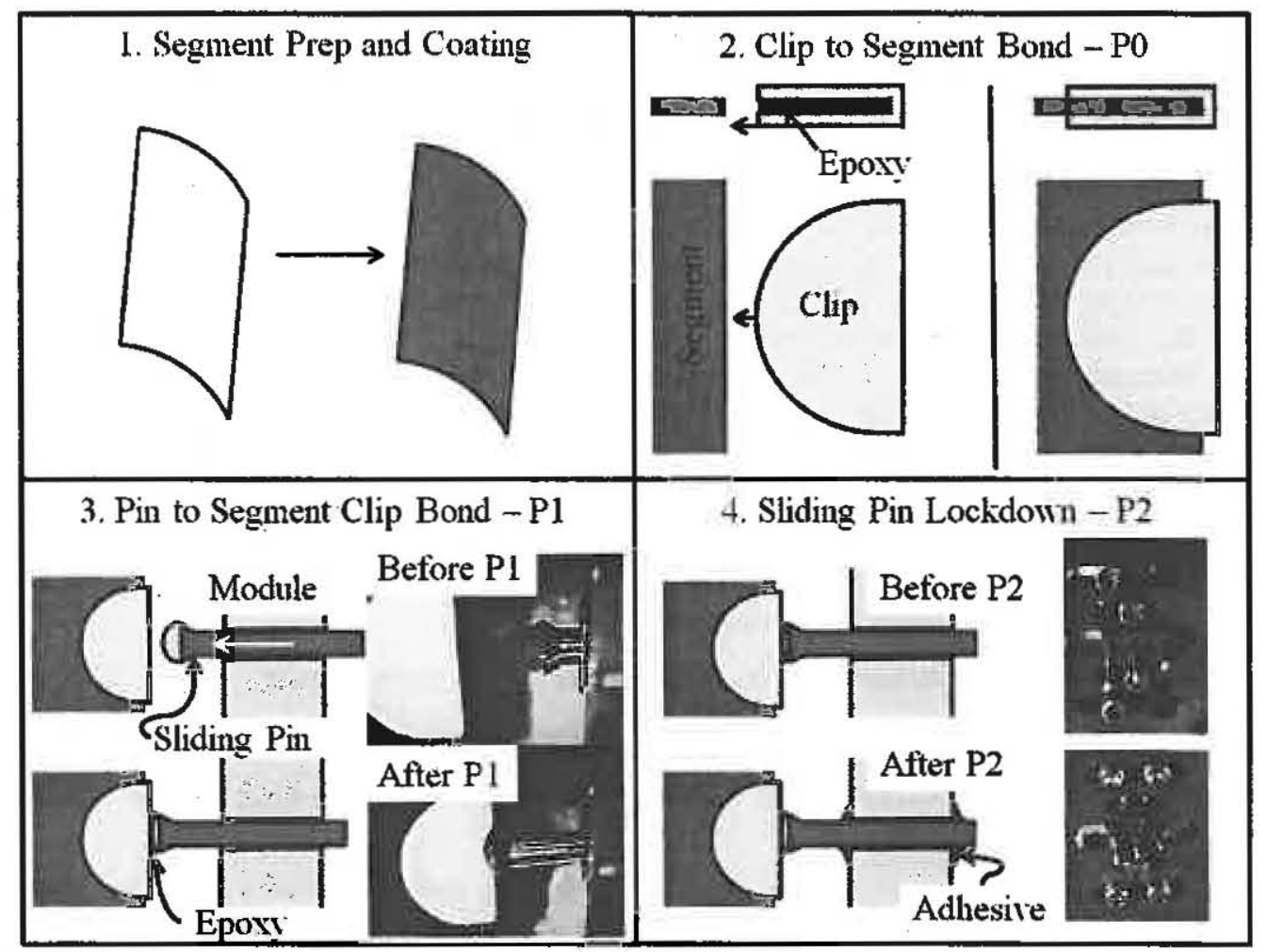

Figure 3. After each segment is coated, 3 clips on each side of the segment are bonded onto the segment (P0). After the segment is aligned into the module, 6 sliding pins with epoxy contact the clips (P1). The sliding pins are locked down in the module using adhesive (P2). The two-step segment permanent mounting procedure (P1 and P2) allows for the curing of the adhesives to take place in a stable thermal environment with limited sources of heat in close proximity to the segment like lights, cameras, and humans which would thermally distort the segment.

\subsection{Permanent mounting hardware}

Segment clips bonded directly to each segment provide several key benefits such as additional surface area for the P1 bond. The clip surface can be treated for multiple bond attempts enabling the same segment to be permanently mounted multiple times. The clips help prevent degrading of the segment integrity over time by limiting the introduction of additional mirco-cracks along the edge of the mirror during segment cleaning and bond surface preparation that could propagate and cause segment failure.

Sliding pins bond directly to the segment clips during the P1 bond process. The sliding nature of the pins allow for precise $\mathrm{P} 1$ bond gap control during the wetting process. At this point in the segment production and permanent mounting preparation there are slight variations in the relative distance from the module wall to the outer bond surface of each segment clip. The pins that were used to permanently mount segments have come in a range of sizes and shapes. Recently pins with a larger diameter tip were used to increase the bond area for P1 in an attempt to improve segment alignment stability. Each pin slides through a precision hole of a sliding tab that is bolted down to the module wall. The clearance between the pin and the tab hole is minimized to prevent out of axis movements of the pin which would most likely cause radial movements of the segment. Segment alignment is extremely sensitive to radial movements which makes the pin to tab hole clearance a critical factor during segment permanent mounting.

The sliding tabs that the pins and therefore the segments mount to provide an additional degree of adjustability to ensure that the pins align to the center of the segment clips. The segment alignment process has improved over time to the point where this additional degree of adjustability is no longer required and future module designs no longer include sliding tabs. Fixed holes in the module walls will eventually replace the adjustable tabs, thereby simplifying the permanent mounting process by eliminating tab alignment. 
Currently two identical mini-modules capable of mounting three segment pairs each allow the segment alignment and permanent mounting process to be developed and then tested in X-ray to verify the alignment results. Both mini-modules (named Technology Demonstration Modules TDM3 and TDM4) are machined from Kovar $\left(6.7 \mathrm{ppm} /{ }^{\circ} \mathrm{C}\right)$ to roughly match the coefficient of thermal expansion ( $\left.\mathrm{C}^{\prime} \mathrm{TE}\right)$ of the glass used for the segments $\left(6.3 \mathrm{ppm} /{ }^{\circ} \mathrm{C}\right)$.

\subsection{Edge-bond procedure}

After a chosen segment with clips attached has been aligned in the module as described in Sections 2.4 and 2.5, the edge-bond permanent mounting process is initiated. A mixed two-part epoxy is applied to the tips of the pins, and each pin is pushed in towards the segment clip until the desired bond gap is achieved. A closed loop visual feedback system is used to determine how far each pin is inserted. A camera with adjustable zoom and focus magnifies the pin tip, adhesive, and clip onto a large display monitor. The pin is pushed in with a nano-actuator capable of steps on the order of $30 \mathrm{~nm}$, $12.5 \mathrm{~mm}$ travel and up to $50 \mathrm{~N}$ of force. The nano-actuator is controlled manually via a rotating knob which in turn controls the approach speed and position of each pin. Final adjustments to segment alignment are made just prior pushing in the first pin but after the first pin contacts the respective segment clip no more alignment adjustments are made

After the final pin is pushed into position, P1 bonding is complete and P2 bonding begins. A low viscosity anaerobic adhesive is used for P2 that flows into the small clearance between the pin and the tab hole. Once adhesive is applied to all pins P2 is complete. Thermal insulation is installed around the entire structure soon after the application of the adhesive is complete. At that time any unnecessary sources of heat such as lights, cameras, and humans are removed from the thermal environment immediately surrounding the segments and module during the curing process. After $18 \mathrm{hrs}$ both P1 epoxy and P2 adhesive is cured to the point that the temporary segment mount can be simply lowered away since only gravity kept the segment in place in the kinematic mount. The temporary balls attached to the top and bottom edge of the segment used to hold the segment in the mount are also removed from the now permanently mounted segment.

A typical work day schedule for permanently mounting segments starts out with lowering the temporary mount from the segment permanently mounted the previous day (Segment A) and removing the temporary balls from that segment. The next segment to be permanently mounted (Segment B) and the associated hardware is prepared and Segment B is seated into the temporary kinematic mount. Segment B is typically aligned shortly after midday and permanent mounting is completed during the early afternoon. Hartmann measurements are collected throughout the alignment and permanent mounting process as wells as overnight to track alignment as the P1 and P2 adhesives harden. The process repeats the following morning with temporary mount removal for Segment B.

\section{ALIGNMENT AND PERMANENT MOUNTING RESULTS}

\subsection{Alignment reaults In optlcal light}

Before, during and after each segment is aligned and permanently mounted into a module Hartmann measurements are taken at least once per hour. Charts similar to that shown in Figure 4 are generated for each segment that is permanently mounted in a module. If more than one segment is permanently mounted in a module the co-alignment between each pair can also be tracked over time (see Figure 5 for an example).

As of June 2012, six mirror segments were aligned and permanently mounted into a module four times. Improvements to the alignment and segment mount procedure were implemented for each of the four completed modules. Most improvements yielded shorter module completion times, better segment pair alignment, and/or better co-alignment between multiple segment pairs. Figure 6 shows a summary of the performance of the four completed modules. Significant progress has been made in just four complete iterations. Improvements to the P1 and P2 procedures and better control of the pin/tab clearance have dramatically improved the average segment pair alignment HPD to less than 5 arcsec. Also the time spent aligning and bonding a single segment has improved from 3.8 to almost 1.0 work days per segment. By meeting the 1.0 work days per segment requirement there can be rapid development of the permanent mounting process with nearly immediate feedback. 


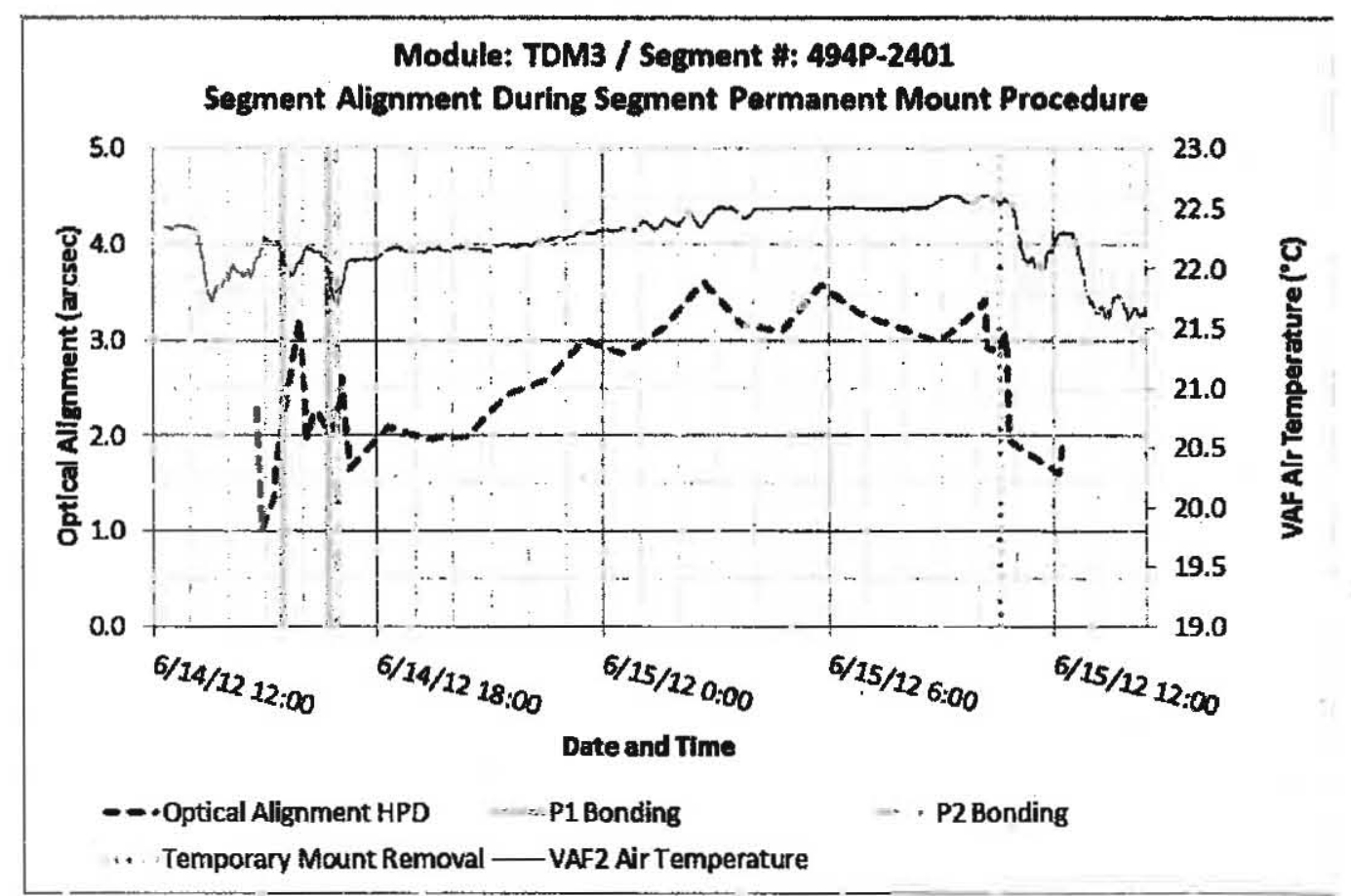

Figure 4. Segment alignment is tracked during permanent mounting using Hartmann measurements in optical light which report the alignment as the segment half power diameter (HPD) in arc seconds. There is a clear correlation with the air temperature near the segment during the segment permanent mount procedure.

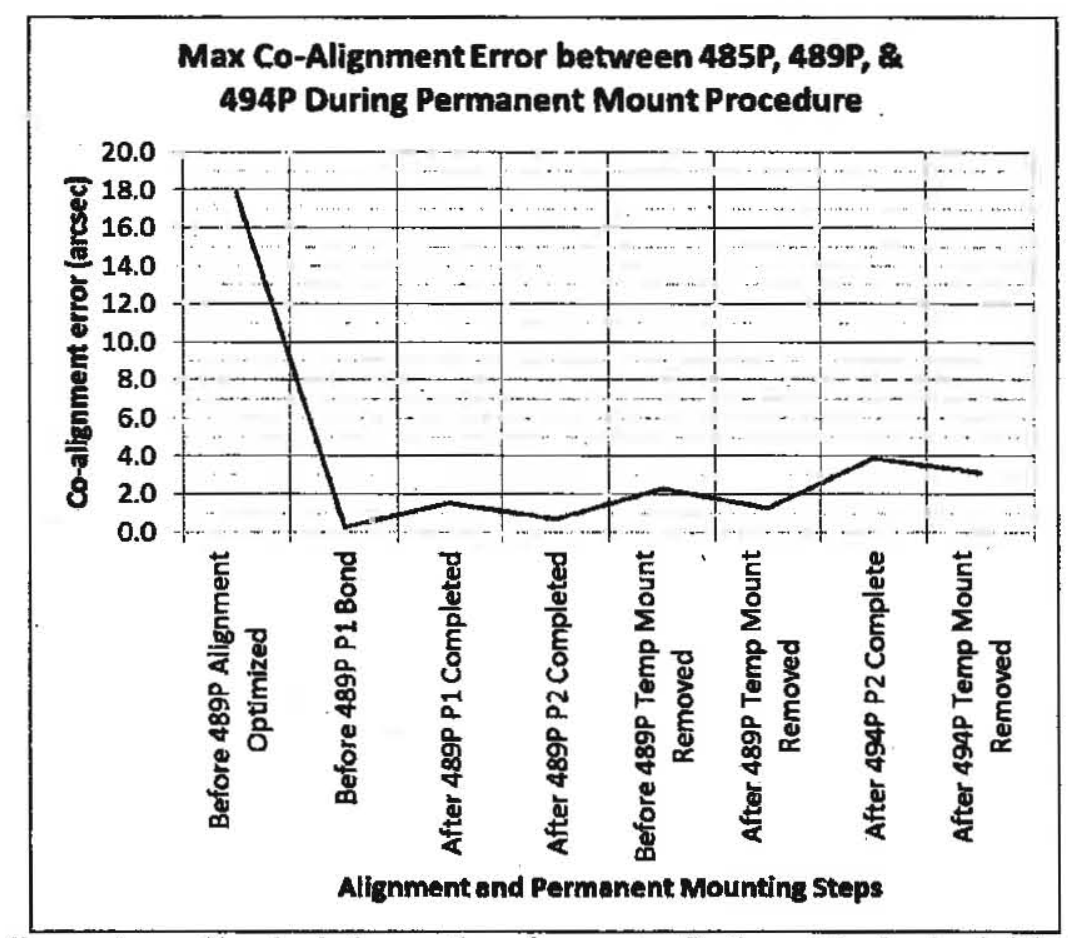

Figure 5. Co-alignment error (the physical separation of segment reflections at the focal point) is tracked as more than one segment is aligned and permanently mounted into a module. A large co-alignment error $(>5$ arcsec) will negatively affect the eventual three pair X-ray resolution. Note: 485,489 , and 494 indicate mirror diameter in millimeters. The "P" indicates that the segments shown in this plot were primary mirror segments. 


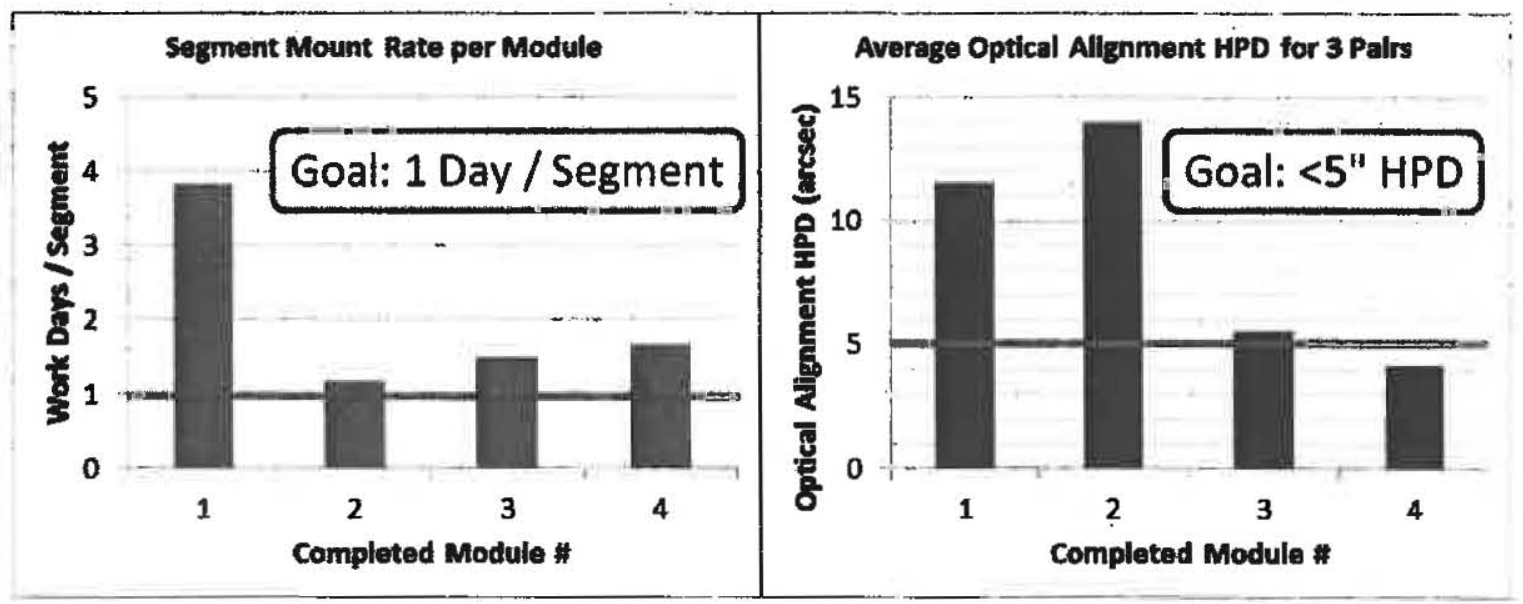

Figure 6. To date, modules containing three pairs of segments aligned and permanently mounted into place were completed four times. Each completed module used new variations of the alignment and permanent bond procedure which resulted in an overall improvement in segment mount rate (left chart) and overall post-permanent mount segment pair alignment quality (right chart).

\subsection{Verification of three segment pair alignment in X-ray}

Once a module has been populated with three segment pairs that were aligned using optical light, the three pair performance is measured in X-ray at the $600 \mathrm{~m}$ X-ray beam line located at the Goddard Geophysical and Astronomical Observatory (GGAO) in Greenbelt, MD. A titanium (4.5 keV) or chromium (5.4 keV) target is located at one end of the evacuated tube and serves as the $\mathrm{X}$-ray source, while the completed module is placed in a vacuum chamber located at the other end of the $600 \mathrm{~m}$ tube (see Figure 77. $8.5 \mathrm{~m}$ beyond the vacuum chamber that houses the module, an X-ray CCD is positioned at the focal point of the mirror segments. Figure 8 shows the three pair combined X-ray result collected for the last completed module.

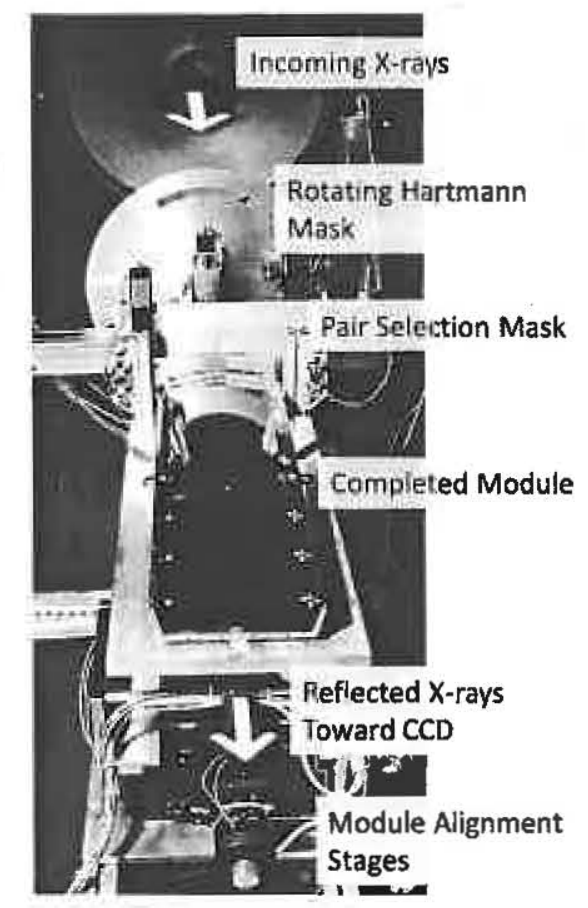

Figure 7. The X-ray test configuration inside the vacuum chamber is located at the end of a $600 \mathrm{~m}$ long X-ray beam line at Goddard Geophysical and Astronomical Observatory (GGAO) housing the permanently mounted segments in the module. The module is resting on a kinematic baseplate that can be adjusted in four degrees of 
freedom. Masking in front of the mirror segments allow for individual segment pairs to be selected. Reflected Xrays travel a further $8.5 \mathrm{~m}$ from the module to the X-ray CCD (not pictured).
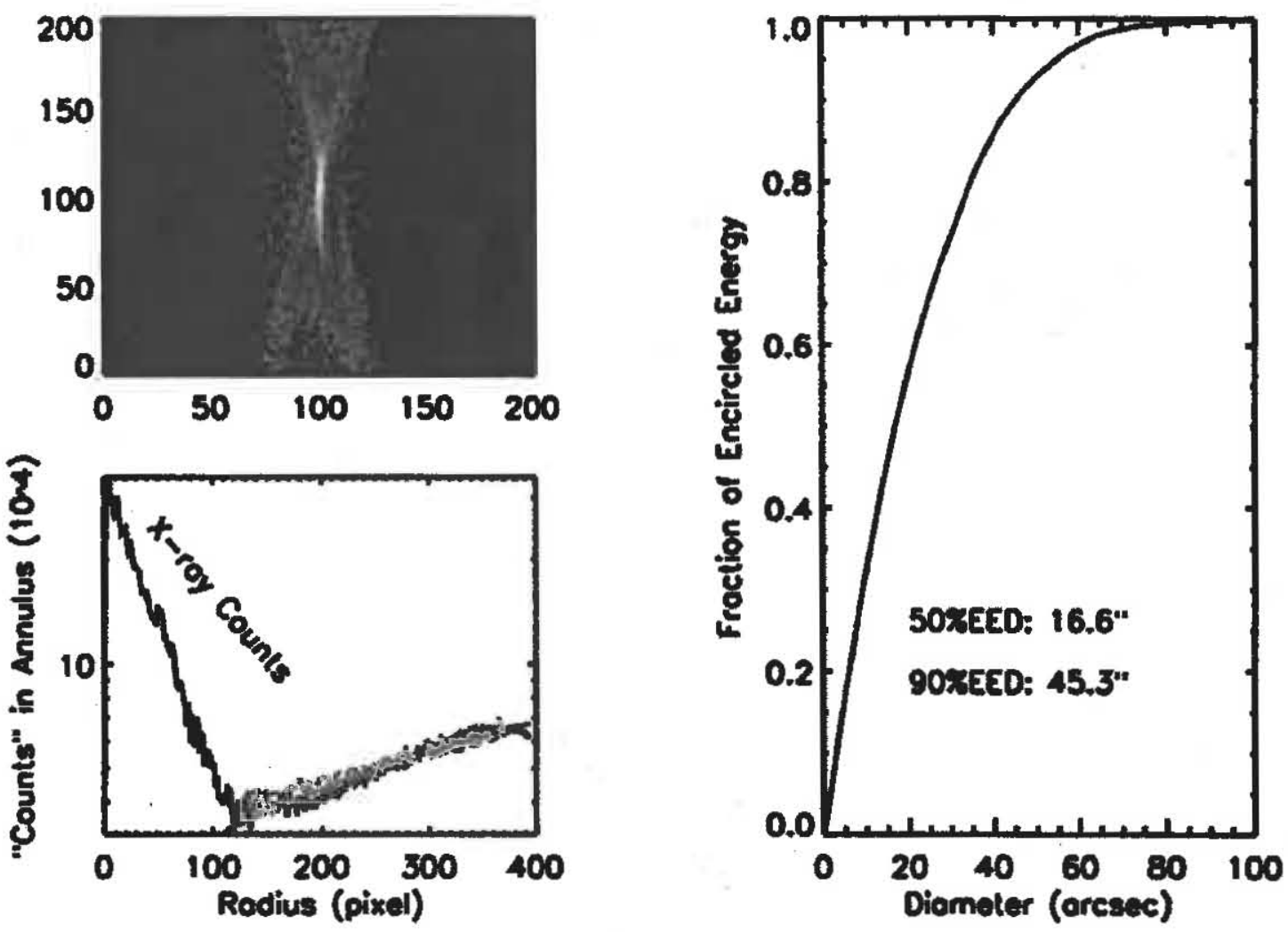

Figure 8. The X-ray test result for three pairs of segments permanently mounted in completed module \#4 was 16.6 arcsec HPD. Segment pairs of $485 \mathrm{~mm}, 489 \mathrm{~mm}$, and $494 \mathrm{~mm}$ in diameter were used.

\section{FUTURE ALIGNMENT AND PERMANENT MOUNTING WORK}

\subsection{Next 6 Months}

Building off of recent successful X-ray tests performed for three segment pairs, improving the three pair angular resolution will be the next major milestone. To accomplish this particular task there are several challenges to overcome first.

After the segments permanently mounted into a module are subjected to a vacuum for X-ray testing, there is a permanent change in the segment alignment. Optical alignment HPD calculated from Hartmann measurements before and after subjecting the completed modules to vacuum show on average a 3-10 arcsecond increase in individual segment HPD. The most likely cause for a permanent change in alignment in vacuum is outgassing of the adhesives used to permanently mount the segments. Several possible low-outgassing replacement adhesives have been identified and are scheduled to be tested shortly.

Improving the thermal environment surrounding the module during segment alignment and permanent mounting is also a high priority. The relative position of the reflection on the CCD located at the focal point tracks closely with the air temperature near the module (see Figure 9). The Vertical Alignment Facility (VAF) that comprises of the collimated red light source, module populated with segments, kinematic module stand, hexapods and associated mounts are all enclosed with rigid thermal insulating foam boards. The foam boards limit airflow into the enclosed VAF when installed except through airflow controlled inlets. The foam boards also block radiative heat transfer from sources external to the VAF. The foam insulating boards are only removed if access to the module is required, during adhesive curing the boards 
remain installed to prevent changes in the air temperature greater than $\pm 0.2 \mathrm{deg} C$. The room temperature external to the $\mathrm{VAF}$ is currently controlled to $\pm 0.5 \mathrm{deg} \mathrm{C}$ which can lead to a substantial difference in air temperature inside the VAF compared to external room air temperature. During the alignment and permanent mounting procedure, the rigid foam boards are removed and installed several times at which point quick changes in air temperature inside the VAF could affect the position of the reflection for mounted segments and segments in the process of being mounted alike. Air temperature change in the VAF is a likely influence on the co-alignment of newly mounted segments. There are several improvements to the thermal stability control that are currently under investigation.

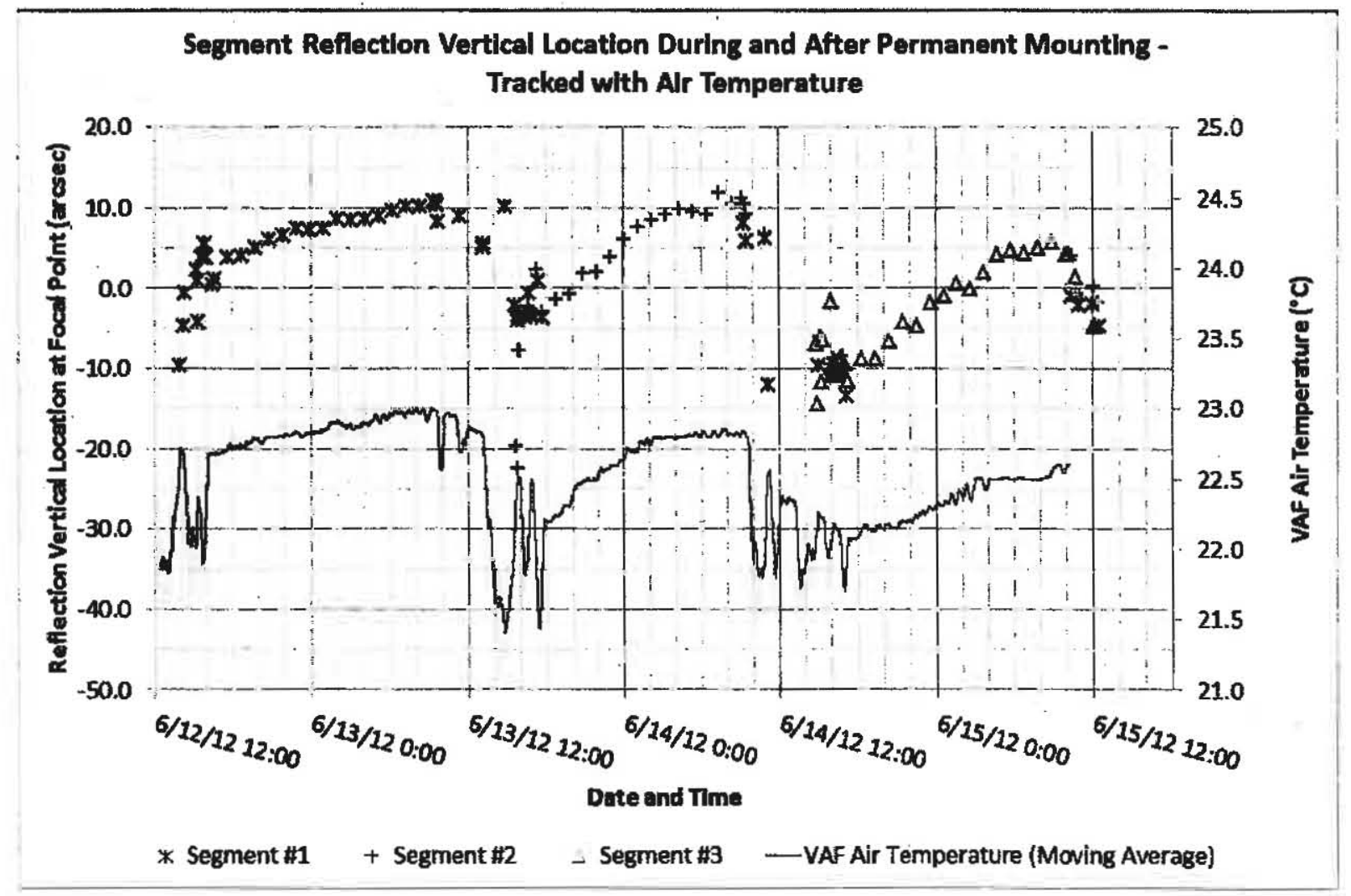

Figure 9. Air temperature sensitivity for the vertical reflection locations for three segments during the alignment and permanent mounting process at the focal point is shown in the above chart over a period of 73 hours. Image centroids obtained using a CCD camera located at the focal point are used to calculate the relative vertical location of the segment reflections.

Along with improving three pair resolution, the first set of vibration tests will be conducted to verify the stability of the segment permanent mount. Any detected weaknesses in the permanently mounted segments will be identified and the segment permanent mount procedure will be improved. In parallel with module vibration testing, analysis tools will be further improved to closely match the conditions that permanently mounted segments are subjected to.

Software control of segment alignment and permanent mounting is currently designed for single segment pairs and has limited built in analysis tools. Segment alignment data processing currently accounts for roughly $1 / 4$ of the total time needed to permanently mount a segment. Automatic data processing along with automatic handling of data from multiple segment pairs will significantly reduce alignment and permanent mounting duration as well as add additional segment alignment functionality. 


\subsection{Next $18 M$ onths}

Aligning and permanently mounting three pairs of segments in a module that is subject to full environmental testing (vibration testing including random vibration, sine sweep, acoustic testing, in addition to thermal cycling, vacuum, etc.) and meets the 10 arcsec HPD at $4.5 \mathrm{keV} \mathrm{X}$-ray test requirement is the top goal for permanent mounting work for the next 18 months. The alignment and permanent segment mount procedure used to meet this requirement must also be repeatable and completed within six working days or less (one working day to align and permanently mount each mirror segment). A system that is more automated than mentioned in the previous section may prove to be necessary to reach 10 arcsec HPD repeatedly. As the segment alignment and permanent mount hardware and procedure matures over time the path towards an automated process becomes clearer. Automation introduces other benefits such as improved reliability, repeatability, and speed.

\section{CONCLUSIONS}

The first steps towards assembly of modules under 10 arcsec show encouraging results in X-ray for three segment pairs at this early stage. One clear factor that directly affects the success of the alignment and permanent segment mounting is the ability to quickly identify areas in need of improvement, determine and implement the necessary changes, and verify through experimentation. One of the short term goals in alignment and permanent segment mounting is to maintain a two week module assembly and X-ray test schedule. Testing new hardware or procedural changes in two week cycles allows for more flexibility and the ability to fail ideas fast to keep resources available (such as our one of a kind Vertical Alignment Facility).

\section{REFERENCES}

[1] Fabian, A. C., "1H0707-495 in 2011: an X-ray source within a gravitational radius of the event horizon," Mon. Not. R. Astron. Soc. 419, 116-123 (2012).

[2] Zhang, W. W., "Next generation x-ray optics: high-resolution, lightweight, and low-cost," Proc. SPIE 8443, (2012).

[3] McClelland, R. S., "Design and analysis of modules for segmented glass x-ray optics," Proc. SPIE 8443, (2012). 